\title{
Prevención farmacológica de embolia por fibrilación auricular y sus escalas de riesgo de embolia y sangrado
} CrossMark

\section{Pharmacological prevention of embolism in atrial fibrillation and their risk scales for embolism and bleeding}

\author{
Enrique Melgarejo-Rojas ${ }^{a, b, c, d}$ \\ a Presidente, Sociedad Colombiana de Cardiología y Cirugía Cardiovascular, Bogotá, Colombia \\ b Hospital Militar Central, Bogotá, Colombia \\ c Colegio Panamericano del Endotelio, Bogotá, Colombia \\ d Comité de Electrofisiología no Invasiva, Colegio Colombiano de Electrofisiología, Bogotá, Colombia
}

Recibido el 3 de octubre de 2016; aceptado el 6 de octubre de 2016

Disponible en Internet el 11 de noviembre de 2016

\section{¿Qué se entiende por fibrilación auricular valvular?}

Acorde con las Guías Europeas de fibrilación auricular de la Sociedad Europea de Cardiología -actualización 2012 ${ }^{1}$, el término "fibrilación atrial valvular" se usa para determinar aquella que está correlacionada con enfermedad reumática valvular (predominantemente estenosis mitral modera a severa) y las válvulas protésicas mecánicas.

\section{Escalas de riesgo de embolia}

La fibrilación auricular se conoce como la arritmia del siglo XXI debido a su creciente prevalencia e incidencia, simplemente por aumento de la supervivencia y disminución de la mortalidad por causas cardiovasculares en la especie humana. Como consecuencia, en el mundo hoy existen más personas de edad avanzada que hace 50 años y por consiguiente más casos de enfermedad hipertensiva o coronaria, que a su vez son causas preponderantes para fibrilación

Correo electrónico: enrique.melgarejo@gmail.com auricular por mecanismos de aumento de la presión intraatrial, fibrosis o dilatación por sobrecarga de presión o de volumen. Aunado a lo anterior, la fibrilación auricular es la arritmia -y la entidad- más generadora de cardioembolia, de ahí que todas las guías coincidan en que el manejo fundamental, el pivote de la terapéutica -independientemente de la restauración del ritmo o del control de la frecuencia cardíaca- debe ser la anticoagulación para prevenirla.

Desde hace 62 años la única alternativa para tal fin era la warfarina, con sus riesgos y complicaciones, a pesar de su efectividad. Dentro de sus inconvenientes está el temido ataque cerebro-vascular hemorrágico, lo cual creó la cultura de anticoagular con cierto "temor", y/o utilizar antiplaquetarios con el fin de "hacer algo" en lugar de nada. La warfarina -como se verá más adelante-, inhibe tres factores de coagulación simultáneamente, que la hacen muy eficaz pero a su vez causante de mayor riesgo de sangrado y no siempre es fácil controlar su estrecho rango terapéutico, aunado a la necesidad de controles casi semanales del INR con los costos e interferencia en la calidad de vida que esto implica. En estudios controlados en los cuales hay un seguimiento estricto de los enfermos medicados con warfarina para el control óptimo del INR, el porcentaje de pacientes dentro del rango terapéutico adecuado (entre 2 y 3 del INR) 


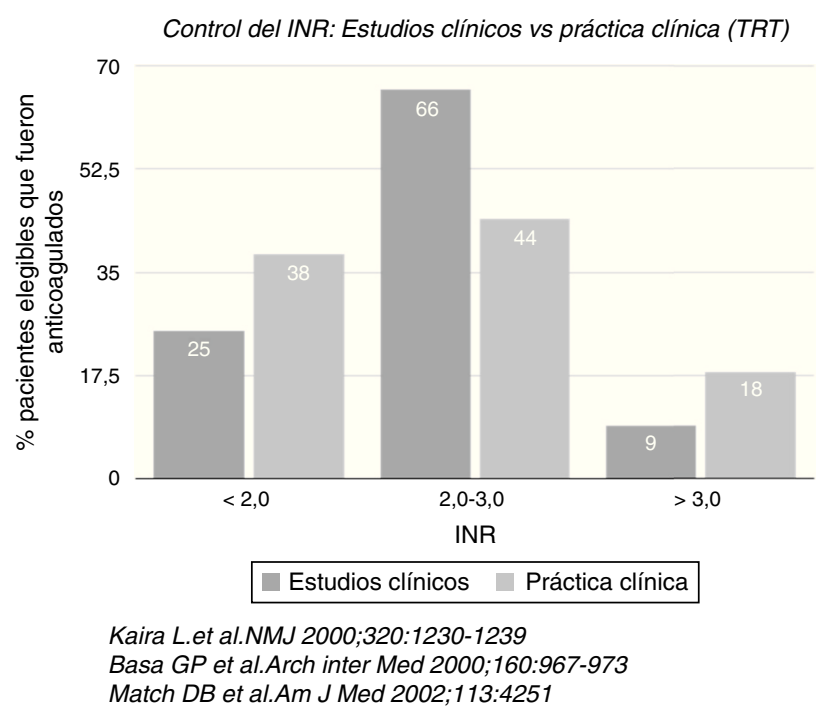

Figura 1 Control del INR en estudios clínicos vs. vida real.

se obtuvo en un $66 \%$, pero en la vida real, se llegó escasamente a un $44 \%$ (fig. 1) y comparativamente, hay mayor tendencia a estar en el rango subterapéutico (riesgo de cardioembolia) que en el de sobreanticoagulación (riesgo de hemorragia). Esta es la incertidumbre de anticoagular.

Los hechos han cambiado en los últimos ocho años; ahora se cuenta con nuevos anticoagulantes no vitamina $K$ dependientes (los denominados DOAC, su sigla en inglés por Direct Oral Anti-Coagulants) y con escalas de riesgo para tratar de predecir cardioembolia en fibrilación auricular o para calcular el riesgo de sangrado por anticoagulación, elementos que han facilitado el control de estas variables con menos incertidumbre.

\section{Escalas de riesgo para ataque cerebro-vascular en pacientes con fibrilación auricular no valvular}

Para la mayoría de quienes padecen fibrilación auricular, actualmente existen múltiples guías (europeas, NICE, canadienses, AHA/ACC, Chest Physicians, regionales y locales), que aplicadas en la vida cotidiana, todas, sin excepción, recomiendan anticoagular, pero también establecen cuando no hacerlo, como es el caso de enfermos con fibrilación auricular aislada -un evento de fibrilación auricular paroxísticaen menores de 65 años, puesto que la evidencia ha mostrado "riesgo muy bajo"'. No obstante, existe una diferencia entre sexo y pronóstico: una mujer de 65 años o más con un factor de riesgo ${ }^{1}$, deberá recibir anticoagulación, incluso si tiene fibrilación auricular aislada ${ }^{2,3}$. Entre las escalas, a la fecha existen dos validadas: la $\mathrm{CHADS}_{2}$ y la $\mathrm{CHA}_{2} \mathrm{DS}_{2}$-VASc.

\section{Escala $\mathrm{CHADS}_{2}$}

Esta tiene en consideración la falla cardíaca congestiva, la hipertensión, la edad $\geq 75$, la diabetes y el ataque cerebrovascular (doble valor) (tabla 1). Es simple, pero no incluye otros factores de riesgo para ataque cerebro-vascular y su limitación ya ha sido cuestionada y discutida, tema al cual se
Tabla 1 Puntaje de $\mathrm{CHADS}_{2}$ y valor predictivo de acuerdo con puntaje obtenido

\begin{tabular}{lll}
\hline $\begin{array}{l}\text { Pacientes } \\
(\mathrm{n}=1.733)\end{array}$ & $\begin{array}{l}\text { Tasa ajustada de ataque } \\
\text { cerebro-vascular \%/año. IC } \\
95 \%\end{array}$ & CHADS $_{2}$ \\
\hline 120 & $1,9(1,2-3,0)$ & 0 \\
463 & $2,8(2,0-3,8)$ & 1 \\
523 & $4,0(3,1-5,1)$ & 2 \\
337 & $5,9(4,6-7,3)$ & 3 \\
220 & $8,5(6,3-11,1)$ & 4 \\
65 & $12,5(8,2-17,5)$ & 5 \\
5 & $18,2(10,5-27,4)$ & 6 \\
& & \\
\hline Puntaje CHADS & & 2 \\
\hline Ataque cerebro-vascular previo o ataque & 1 \\
$\quad$ isquémico transitorio & 1 \\
Edad $>$ 75 años & \\
Hipertensión & 1 \\
Diabetes & \\
Falla cardíaca & \\
\hline
\end{tabular}

suma el que su antigüedad ya tiene dos décadas y fue aplicada en los estudios iniciales con DOAC. En estos ensayos no se consideró el impacto progresivo de la edad, y hoy día se sabe que el riesgo para ataque cerebro-vascular es más alto en la población mayor de 65 años e incrementa progresivamente a medida que lo hace la edad; adicionalmente, tampoco se tuvo en cuenta el antecedente de cualquier enfermedad vascular, que también eleva el riesgo de embolia. Así mismo, la función renal queda en el vacío ya que a mayor deterioro, mayor aumento del riesgo de sangrado ${ }^{4}$.

Pero obsérvese (tabla 1) que muchos enfermos clasificados como de "bajo riesgo" con el $\mathrm{CHADS}_{2} \quad($ score $=0)$ tienen una tasa de eventos de ataque cerebro-vascular de 1,9\%/año, con intervalos de confianza de 1,2-3,0, es decir, hay un "gap", que puede ser importante para esa población subestimada ${ }^{5,6}$. La tendencia actual es identificar enfermos de bajo riesgo real más que los de alto riesgo. Se debe ser más inclusivos que exclusivos con los factores de riesgo para ataque cerebro-vascular, si bien todo paciente con un valor de 1 o mayor deberá ser anticoagulado, principalmente con $\mathrm{DOAC}^{7}$.

\section{Escala $\mathrm{CHA}_{2} \mathrm{DS}_{2}$-VASc}

Esta es la escala más recomendada por todas las guías actuales, aunque ha habido propuestas de nuevos scores, como por ejemplo el SAMe-TT2R2, que tiene la ventaja de tomar en consideración el tiempo dentro del rango terapéutico del INR ${ }^{8}$. La escala $\mathrm{CHA}_{2} \mathrm{DS}_{2}-\mathrm{VASC}$ ha sido validada en múltiples estudios y tiene la fortaleza de incluir otros factores de riesgo determinantes de riesgo para ataque cerebrovascular $^{9}$. En la tabla 2 se comparan las diferencias entre estas dos escalas.

El $\mathrm{CHA}_{2} \mathrm{DS}_{2}$-VASc ya ha evidenciado ser una herramienta superior para identificar "verdaderos casos de bajo riesgo" ${ }^{10-13}$ (tabla 3 ). Es, además, más sensible con relación a la $\mathrm{CHADS}_{2} 6,14,15$. 
Tabla 2 Diferencias entre las escalas $\mathrm{CHADS}_{2}$ y $\mathrm{CHA}_{2} \mathrm{DS}_{2}$ VASc

\begin{tabular}{|c|c|}
\hline \multicolumn{2}{|l|}{ Escala $\mathrm{CHADS}_{2}$} \\
\hline $\begin{array}{l}\text { Ataque cerebro-vascular previo o ataque } \\
\text { isquémico transitorio }\end{array}$ & 2 \\
\hline Edad $>75$ años & 1 \\
\hline Hipertensión & 1 \\
\hline Diabetes & 1 \\
\hline Falla cardíaca & 1 \\
\hline \multicolumn{2}{|c|}{$0=1,9 \%, 1=2,8 \%, 2=4 \%, 3=5,9 \%, 4=8,5 \%, 5=12,5 \%, 6=18 \%$} \\
\hline \multicolumn{2}{|l|}{$\mathrm{CHA}_{2} \mathrm{DS}_{2}-\mathrm{VASC}$} \\
\hline Falla cardíaca/disfunción ventricular izquierda & 1 \\
\hline Hipertensión & 1 \\
\hline Edad $\geq 75$ años & 2 \\
\hline Diabetes mellitus & 1 \\
\hline $\begin{array}{l}\text { Ataque cerebro-vascular/accidente isquémico } \\
\text { transitorio/tromboembolia }\end{array}$ & 2 \\
\hline Enfermedad vascular (C, Car, P, Ao) & 1 \\
\hline Edad $65-74$ & 1 \\
\hline Sexo femenino & 1 \\
\hline \multicolumn{2}{|c|}{$\begin{array}{l}0=0 \%, 1=1,3 \%, 2=2,2 \%, 3=3,2 \%, 4=4 \%, 5=6,7 \%, 6=9,8 \%, \\
7=9,6 \%, 8=6,7 \%, 9=15,2 \%\end{array}$} \\
\hline
\end{tabular}

En la población china, el riesgo de ataque cerebrovascular con valor de 0 es extremadamente alto (para Taiwán es de $1,06 \%$ y para Hong Kong de 2,41\%). Aun no es claro si se trata de un problema esencialmente étnico o genético que deba ser extrapolado a la población china en general, pero amerita tenerlo en consideración ${ }^{16}$. El $\mathrm{CHA}_{2} \mathrm{DS}_{2}$-VASc también ayuda a definir el riesgo de ataque cerebro-vascular en enfermos después de ablación y deberá aplicarse, pues persiste el riesgo de cardioembolia ${ }^{17}$. Un hecho para tener en cuenta son los pacientes con alteración de la función renal, ya que este es un proceso dinámico y progresivo que en algunos casos puede ser reversible (uso de AINE o fibratos o uso prolongado de inhibidores de la bomba de protones, entre otros); están en mayor riesgo para ataque cerebro-vascular y para mortalidad por eventos coronarios e incluso poseen

Tabla 3 Valor predictivo del $\mathrm{CHA}_{2} \mathrm{DS}_{2}$-VASc para bajo y alto riesgo (desde $0 \%$ hasta 15,2\%)

\begin{tabular}{lcl}
\hline $\begin{array}{l}\text { Pacientes } \\
(\mathrm{n}=7.329)\end{array}$ & $\begin{array}{l}\text { Tasa ajustada de } \\
\text { ataque } \\
\text { cerebro-vascular } \\
\text { \%/año }\end{array}$ & $\mathrm{CHA}_{2} \mathrm{DS}_{2}$-VASc \\
\hline 1 & 0 & 0 \\
42 & 1,3 & 1 \\
1.230 & 2,2 & 2 \\
1.730 & 3,2 & 3 \\
1.718 & 4,0 & 4 \\
1.159 & 6,7 & 5 \\
679 & 9,8 & 6 \\
294 & 9,6 & 7 \\
82 & 6,7 & 8 \\
14 & 15,2 & 9 \\
\hline
\end{tabular}

mayor riesgo de sangrado. En consecuencia, deberán evaluarse estos cambios de riesgo en forma periódica mediante el $\mathrm{CHA}_{2} \mathrm{DS}_{2}$-VASc, para así determinar cambio de terapia (si progresa a falla renal severa con TFG por debajo de 30 , se opta por la warfarina).

\section{¿Cuándo usar $\mathrm{CHA}_{2} \mathrm{DS}_{2}$-VASc?}

- En todo tipo de fibrilación auricular o flutter atrial.

- Cuando existe riesgo de recurrencia después de cardioversión.

- Una vez establecido el riesgo y la decisión de anticoagular, se deberá evaluar el riesgo de sangrado mediante la escala de HAS-BLED (ver más adelante), para establecer la relación beneficio de prevenir ataque cerebro-vascular embólico, vs. sangrado mayor como complicación. De manera concomitante se hace necesario modificar los siguientes factores de riesgo: hipertensión arterial no controlada, uso de Aspirina y AINE -deberán suspenderse-, control de alcoholismo y obesidad, si se tiene INR lábil -riesgos de supra o infraanticoagulación, mayor riesgo de embolia o de hemorragia respectivamente-, será preferible el uso de DOAC.

- Deberá tenerse en cuenta si es un anciano frágil y lábil, con tendencia a caídas o demencia, antes de iniciar anticoagulación, y más si vive solo.

- Establecer el estado cognitivo como elemento de precaución y tener mayor control en estos pacientes, tanto por la parte médica y como la de los familiares..

- Explicarle al paciente los riesgos y beneficios de la anticoagulación, haciéndole saber que en la mayoría de los casos son mayores los beneficios -especialmente con los DOAC- que el riesgo de sangrado. Si el riesgo de sangrado es alto, se requerirá un monitorización estrecha. Por fortuna ya existe y pronto estarán en el mercado los reversores de los DOAC.

- Como ya se mencionó, evaluar periódicamente la función renal (cada 6 meses) en pacientes añosos y/o con función renal comprometida que reciben DOAC.

- Si el $\mathrm{CHA}_{2} \mathrm{DS}_{2}$-VASc. es igual o mayor a 1 , se recomienda el uso de anticoagulantes principalmente el de DOAC.

\section{Recomendaciones con relación al uso de las escalas y sus implicaciones}

Recomendaciones para la prevención de tromboembolias en la fibrilación auricular no valvular (fig. 2) (tabla 4).

Las Guías AHA-ACC de 2014 en lo referente a escalas, recomiendan la $\mathrm{CHA}_{2} \mathrm{DS}_{2}$-VASc.-VASc como única para establecer el riego de ataque cerebro-vascular isquémico o cardioembolia (recomendación I, nivel de evidencia B7). Las Guías NICE, por su parte, recomiendan el uso del $\mathrm{CHA}_{2} \mathrm{DS}_{2}$ VASc y coinciden en gran parte con las europeas, salvo que recomiendan anticoagulación a partir de un puntaje $\mathrm{CHA}_{2} \mathrm{DS}_{2}$-VASc de 2 o superior. No recomiendan uso de Aspirina en ninguna circunstancia ${ }^{18}$. Las Guías canadienses ${ }^{19}$ (CCS) tienen un esquema que difieren un tanto del de las europeas y las americanas. Afirman que la $\mathrm{CHADS}_{2}$ es más fácil y propenden por el uso del algoritmo (fig. 3).

En síntesis, las guías canadienses recomiendan lo siguiente: 
Tabla 4 Recomendaciones y nivel de evidencia de las Guías europeas de cardiología 2012 con base en las escalas $\mathrm{CHA}_{2} \mathrm{DS}_{2}$-VASc y HAS-BLED ${ }^{1}$

\section{Recomendaciones}

Recomendaciones para la prevención de tromboembolias en la fibrilación auricular no valvular: general

Se recomienda tratamiento antitrombótico para prevenir tromboembolias para todos los pacientes con fibrilación auricular, excepto en aquellos (tanto varones como mujeres) en riesgo bajo (edad $<65$ años y fibrilación auricular sola) o con contraindicaciones.

La elección del tratamiento antitrombótico debe basarse en los riesgos absolutos de ataque cerebro-vascular/tromboembolia y hemorragia y en el beneficio clínico neto para el paciente concreto.

La escala $\mathrm{CHA}_{2} \mathrm{DS}_{2}$-VASc. = se recomienda como medio para evaluar el riesgo de ataque cerebro-vascular en la fibrilación auricular no valvular.

Para los pacientes con $\mathrm{CHA}_{2} \mathrm{DS}_{2}$-VASc igual a 0 (es decir, edad < 65 años con fibrilación auricular sola), riesgo bajo y ningún factor de riesgo, no se recomienda ningún tratamiento antitrombótico.

Para los pacientes con $\mathrm{CHA}_{2} \mathrm{DS}_{2}-\mathrm{VASC} \geq 2$, a menos que esté contraindicado, se recomienda el tratamiento anticoagulante con:

- AVK con ajuste de dosis (INR 2-3) o

- Un inhibidor directo de la trombina (dabigatrán) o

- Un inhibidor oral del factor Xa (p. ej. rivaroxabán, apixabán).

Para los pacientes con $\mathrm{CHA}_{2} \mathrm{DS}_{2}$-VASc igual a 1, basándose en una evaluación del riesgo de complicaciones hemorrágicas y las preferencias del paciente, se debe considerar el tratamiento anticoagulante con:

- AVK con ajuste de dosis (INR 2-3) o

- Un inhibidor directo de la trombina (dabigatrán) o

- Un inhibidor oral del factor Xa (p. ej. rivaroxabán, apixabán)

Las mujeres de edad < 65 años y con fibrilación auricular sola (pero con $\mathrm{CHA}_{2} \mathrm{DS}_{2}$-VASc igual a 1 en virtud de su sexo) tienen riesgo bajo y no se debe considerar el tratamiento antitrombótico

Si el paciente se niega a tomar cualquier tipo de anticoagulante (ya sea AVK o NACO), se debe considerar tratamiento antiplaquetario mediante terapia combinada con 75-100 mg de AAS más $75 \mathrm{mg} /$ día de clopidogrel (con lo que hay bajo riesgo hemorrágico) o, menos eficazmente, AAS 75-325 mg/día.

Recomendaciones para la prevención de tromboembolias en la fibrilación auricular no valvular: NACO

Cuando un paciente con fibrilación auricular para el que se recomiendan anitcoagulación no puede utilizar un AVK con ajuste de dosis (INR 2-3) debido a problemas para mantener la anticoagulación terapéutica, efectos secundarios con los AVK o imposibilidad de acudir a la monitorización y el control del INR, se recomienda un NACO:

- Un inhibidor directo de la trombina (dabigatrán) o

- Un inhibidor oral del factor Xa (p. ej. rivaroxabán, apixabán)

En los casos que se recomienda anticoagulación, en lugar de un AVK con ajuste de dosis (INR 2-3) para la mayoría de los pacientes con fibrilación auricular no valvular, según su beneficio clínico neto, se debe considerar un NACO:

- Un inhibidor directo de la trombina (dabigatrán) o

- Un inhibidor oral del factor Xa (p. ej. rivaroxabán, apixabán)

Para los casos que se prescribe dabigatrán, se debe considerar una dosis de $150 \mathrm{mg}$ dos veces al día para la mayoría de los pacientes en lugar de $110 \mathrm{mg}$ dos veces al día, y se recomienda una segunda en caso de:

- Ancianos de edad $\geq 80$ años

- Uso concomitante de fármacos con interacciones (p. ej. verapamilo)

- Riesgo hemorrágico elevado (HAS-BLED $\geq 3$ )

- Deterioro renal moderado $(\mathrm{CrCl} 30-49 \mathrm{ml} / \mathrm{min})$

En el caso de rivaroxabán, se debe considerar una dosis de $20 \mathrm{mg}$ una vez al día para la mayoría de los pacientes en lugar de $15 \mathrm{mg}$ una vez al día, y se recomienda una segunda en caso de:

- Riesgo hemorrágico elevado (HAS-BLED $\geq 3$ )

- Deterioro renal moderado $(\mathrm{CrCl} 30-49 \mathrm{ml} / \mathrm{min})$

Se recomienda una evaluación basal, y regularmente después, de la función renal (mediante $\mathrm{CrCl}$ ) en pacientes que estén iniciando tratamiento con algún NACO; debe realizarse anualmente, pero con mayor frecuencia en pacientes con deterioro renal moderado, cuyo $\mathrm{CrCl}$ se debe evaluar dos a tres veces al año.

Los NACO (dabigatrán, rivaroxabán y apixabán) no están recomendados para pacientes con deterioro renal grave $(\mathrm{CrCl}<30 \mathrm{ml} / \mathrm{min})$

Recomendaciones para la prevención de tromboembolias en la fibrilación auricular no valvular: hemorragia Se recomienda evaluar el riesgo hemorrágico al prescribir tratamiento antitrombótico (ya sea con AVK, NACO, AAS/clopidogrel o AAS solo). 
Tabla 4 (continuación)

\begin{tabular}{l}
\hline Recomendaciones \\
\hline Se debe considerar la escala HAS-BLED como una estimación para evaluar el riesgo hemorrágico, de modo que \\
una puntuación $\geq 3$ indica "alto riesgo"' y hace necesaria cierta preocupación y revisiones regulares tras \\
iniciar el tratamiento antitrombótico, ya sea con anticoagulación o terapia antiplaquetaria (nivel de evidencia \\
A); se deben abordar los factores de riesgo hemorrágico corregibles (p. ej., presión arterial no controlada, INR \\
lábil si el paciente recibe tratamiento con un AVK, fármacos concomitantes [AAS, AINE, etc.], alcohol, etc.) \\
(nivel de evidencia B); se debe utilizar la escala HAS-BLED para identificar los factores de riesgo hemorrágico \\
modificables que deban tratarse, pero no se debe utilizar por sí sola para excluir a los pacientes del \\
tratamiento con anticoagulantes (nivel de evidencia B). \\
El riesgo de hemorragia grave con terapia antiplaquetaria (con terapia combinada de AAS y clopidogrel y, \\
especialmente en ancianos, también con AAS en monoterapia) se debe considerar similar al riesgo con \\
anticoagulación. \\
Recomendaciones para la prevención de tromboembolias en fibrilación auricular no valvular: pericardioversión \\
Para los pacientes con fibrilación auricular $\geq 48$ h o cuando se desconoce la duración de la fibrilación auricular, \\
se recomienda el tratamiento con anticoagulantes (p.ej. AVK con INR 2-3 o dabigatrán) durante al menos tres \\
semanas antes y al menos cuatro semanas después de la cardioversión, independiente del método (eléctrico o \\
con fármacos orales/IV). \\
Para los pacientes con factores de riesgo de recurrencia de ataque cerebro-vascular o fibrilación auricular, el \\
tratamiento con anticoagulantes, ya sea con AVK con ajuste de dosis (INR 2-3) o un NACO, se debería continuar \\
de por vida independiente del mantenimiento aparente del ritmo sinusal tras la cardioversión.
\end{tabular}

1. Todos los pacientes con fibrilación auricular o flutter atrial, sea paroxística o persistente, deben ser estratificados usando un índice predictivo para riesgo de ataque cerebro-vascular (el "algoritmo CCS") basado en el modelo $\mathrm{CHADS}_{2}$ (fuerte recomendación, evidencia de alta calidad).

2. Que la terapia con DOAC sea prescrita para la mayoría de pacientes de o mayores a 65 años o $\mathrm{CHADS}_{2}$ con puntaje 1 (recomendación fuerte, evidencia calidad moderada).

3. Que el ASA $(81 \mathrm{mg} / \mathrm{d})$ sea prescrita para pacientes con ninguno de los riesgos resaltados en el algoritmo CCS (edad< 65 años y no CHADS $_{2}$ y sin factores de riesgo para quienes tienen enfermedad arterial (coronaria, aórtica, o periférica) (recomendaciones condicionales, evidencia moderada).

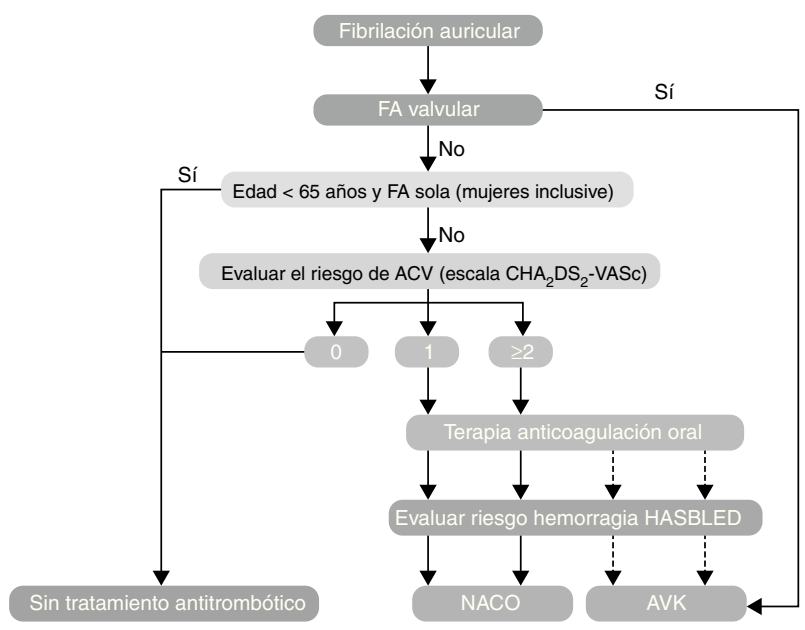

Figura 2 Modificada de: Recomendaciones Guías Europeas de Cardiología $2012^{1}$.

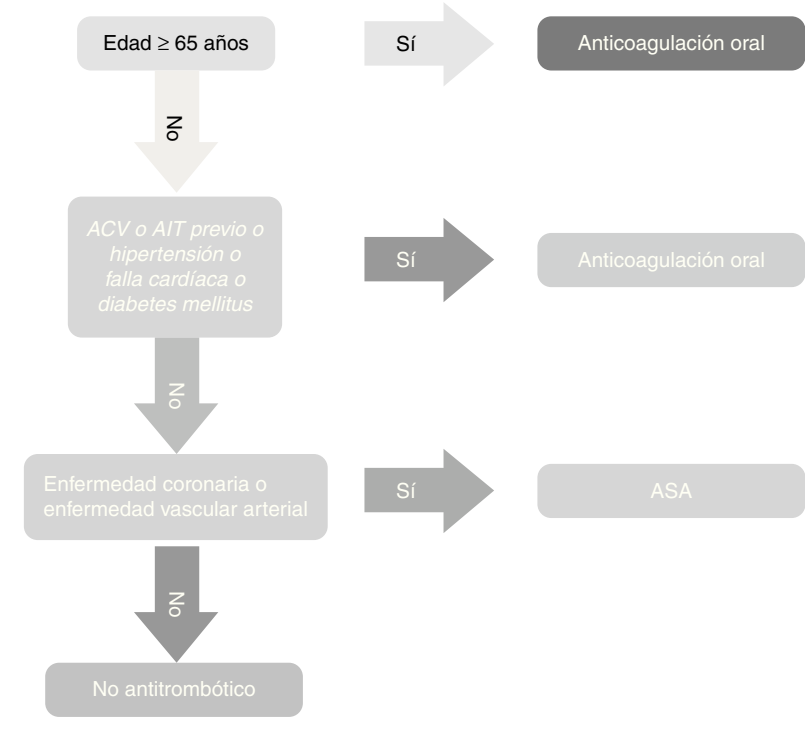

Figura 3 Algoritmo CCS.

4. No prescribir terapia antitrombótica para pacientes con ninguno de los riesgos resaltados en el algoritmo CCS (edad< 65 y no factores de riesgo del $\mathrm{CHADS}_{2}$, y libres de enfermedad vascular arterial (coronaria, aórtica, periférica) (evidencia de baja calidad).

\section{Conclusiones}

Como se aprecia, aun no hay consenso universal para el uso de las escalas, pero la más recomendada actualmente para tomar decisiones es la $\mathrm{CHA}_{2} \mathrm{DS}_{2}$-VASc. 


\section{Escalas de riesgo para sangrado}

Existen varias escalas para estimar el riesgo de sangrado en enfermos con fibrilación auricular que requieran anticoagulación.

La de mayor disponibilidad es la HEMORR ${ }_{2}$ HAGES [Enfermedad Hepática o renal, abuso de Etanol, Malignidad, Older (edad) $\geq 75$ años), Recuento de plaquetas reducido o su función, riesgo de hemorragia (Bleeding risk), Hipertensión (no controlada), Anemia, Factores Genéticos, Exceso riesgo para caídas y Stroke] ${ }^{20}$.

y le sigue otra de uso mayor y más fácil: HAS-BLED [Hipertensión, función renal/hepática Anormal, Stroke, Historia de sangrado o predisposición (Bleeding), INR Lábil, Edad (mayor de 65), Drogas (alcohol, psicotrópicos, etc. $)^{21}$.

También existe la escala ATRIA (AnTicoagulación and Risk factors In AF. ${ }^{22}$

Los diferentes Consensos o Guías referentes a Riesgo de Sangrado de la EHRA, NICE y ESC Working Group on Thrombosis ${ }^{23}$, recomiendan el uso de HAS-BLED, más que otras un tanto complicadas y con menor peso de evidencia $^{24,25}$. El HAS-BLED ha sido valioso en diferentes cohortes independientes ${ }^{14,21,26,27}$ e incluso ha mostrado igual capacidad pronóstica con los enfermos que toman Aspirina o warfarina ${ }^{14}$. De esta manera, se recomienda utilizar simultáneamente $\mathrm{CHA}_{2} \mathrm{DS}_{2}$-VASc y HAS- BLED en todos los enfermos con fibrilación auricular. Un puntaje HAS-BLED $\geq$ 3 , debe poner en alerta para riesgo de hemorragia, además de corregir factores de riesgo potenciales y reversibles para sangrado. El HAS-BLED por sí mismo no debe usarse para excluir pacientes para anticoagulación sino como una herramienta para establecer el riesgo y buscar causas corregibles o un estrecho control del paciente, además de la relación riesgo/beneficio (ataque cerebro-vascular vs. hemorragia intracerebral); Olesen et $\mathrm{al}^{28}$ corroboran su utilidad.

Otros estudios observacionales ${ }^{29}$ con mayor número de casos favoreció el uso de warfarina a pesar de existir riesgo de sangrado, a excepción de la población de muy bajo riesgo para ataque cerebro-vascular isquémico con un $\mathrm{CHA}_{2} \mathrm{DS}_{2}$ VASc de 0 y riesgo moderado a alto para sangrado. Sin embargo, se prefiere el uso de DOAC, al tener menor riesgo de sangrado mayor frente a la warfarina. Solo en los enfermos con riesgo alto de sangrado estaría contraindicado anticoagular.

En conclusión, a la luz de la evidencia actual deberá utilizarse siempre la $\mathrm{CHA}_{2} \mathrm{DS}_{2}$-VASc con el HAS-BLED para determinar riesgo/beneficio de anticoagular enfermos con fibrilación auricular; no obstante, HAS-BLED no debe tomarse como una contraindicación para establecer riesgos y modificarlos. En la tabla 5 se muestran los niveles de evidencia y recomendaciones para HAS-BLED.

\section{¿Cuál es el papel actual de diversos medicamentos?}

\section{Warfarina}

Es un derivado sintético de la cumarina, una sustancia natural que se encuentra en muchas plantas, en particular en la Asperula adorata. En 1950 fue aprobada como medicamento anticoagulante. Su mecanismo de acción se logra al
Tabla 5 Escala HAS-BLED

\begin{tabular}{lc}
\hline & \% Sangrado anual \\
\hline 0 & 1.13 \\
1 & 1.02 \\
2 & 1.88 \\
3 & 3.74 \\
4 & 8.70 \\
5 & 12.5 \\
& \\
HASBLED: riesgo de sangrado & \\
Hipertensión $>160$ mm Hg & 1 \\
Función renal y/o hepática anormal: & \\
$\quad$ Dx, Tx, Cr $>2.3$ / Bil $>2 X$, TA - fibrilación & \\
$\quad$ auricular $>3 X$ & 1 \\
Ataque cerebro-vascular & 1 \\
Historia de sangrado & 1 \\
INR Lábil (<60\% del rango terapéutico) & 1 \\
Edad $>65$ años & 1 ó 2 \\
Drogas (antiplaquetarios y AINE) o abuso de & \\
$\quad$ alcohol & \\
Puntaje $\geq 3:$ alto riesgo de sangrado & \\
\hline
\end{tabular}

inhibir la vitamina $\mathrm{K}$ epóxido-reductasa, una enzima que recicla la vitamina $\mathrm{K}$ a su forma reducida. Actúa inhibiendo simultáneamente los factores de coagulación Ix, Xa y trombina. (fig. 4). A diferencia de los DOAC o los anticoagulantes selectivos que actúan en tan solo un factor de coagulación, se explica de esta manera su menor riesgo para sangrado frente a la warfarina.

Según las Guías de la AHA/ACC del 2007 para terapia con warfarina ${ }^{30}$, la dosis se establece en dos etapas: inicial y de mantenimiento. Después de iniciar el tratamiento, el valor INR se monitoriza con frecuencia hasta obtener una respuesta de dosis estable; después la frecuencia del test de INR se puede reducir a uno cada mes. El efecto anticoagulante se observa entre el segundo al séptimo día

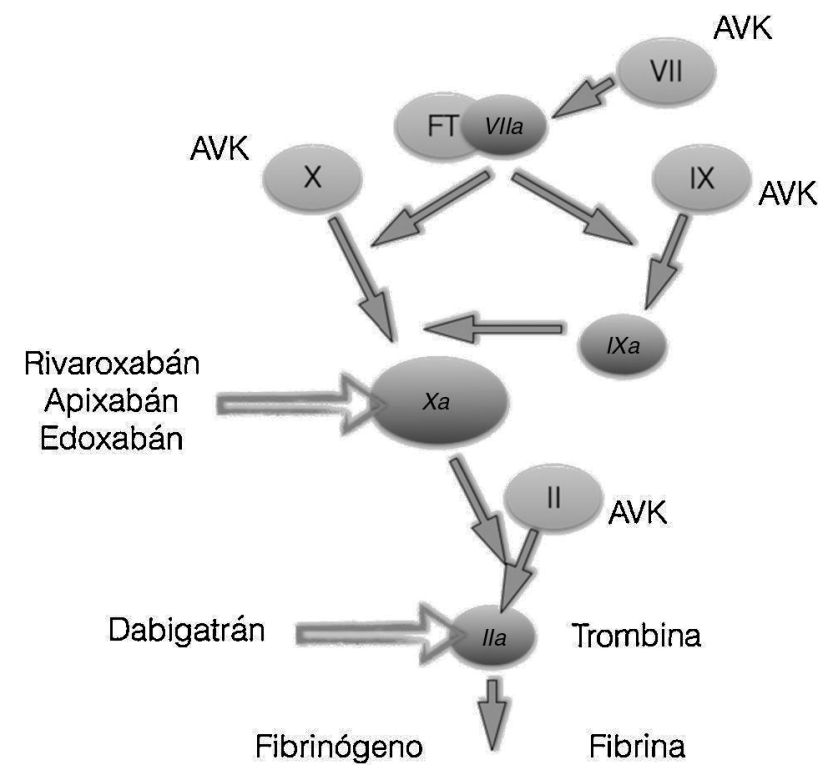

Figura 4 Mecanismos de acción de la warfarina y los DOAC. 
después de iniciar su administración. Cuando se requiere un efecto inmediato, se administra heparina concomitantemente por $\geq 4$ días. La práctica común de administrar una dosis de carga con warfarina generalmente es innecesaria, y hay razones teóricas para comenzar tratamiento con dosis promedio de $5 \mathrm{mg}$ diarios, que realmente resulta en un INR de $\geq 2,0$ después del tercer día. La heparina normalmente puede suspenderse una vez que el INR ha logrado el rango terapéutico. Cuando la anticoagulación no es urgente (ej., fibrilación atrial permanente), se puede comenzar fuera del hospital, con una dosis de $5 \mathrm{mg} / \mathrm{d}$, que usualmente produce un efecto satisfactorio en seis días. En ancianos y/o en aquellos con alto riesgo de sangrado puede iniciarse con una dosis menor. Si no se logran cifras estables, se chequea el uso excesivo o no regularizado de vegetales (ricos en vitamina $\mathrm{K}$ ) o de alcohol, además de la adherencia y persistencia. Por otro lado, es preciso tener en mente los siguientes tópicos:

- La warfarina incrementa el riesgo de ataque cerebrovascular en los pacientes de fibrilación atrial y durante los primeros 30 días de uso.

- El riesgo es particularmente alto durante la primera semana después de que los pacientes empiezan a tomar el medicamento.

- Los pacientes con fibrilación auricular con respuesta ventricular lenta tienen doble riesgo de sufrir ataque cerebro-vascular en los primeros 30 días después de empezar a tomar warfarina, en comparación con quienes no la toman, de acuerdo con un estudio de más de 70.000 pacientes $^{31}$.

- La warfarina está indicada en enfermedad valvular tipo estenosis mitral moderada o severa, y especialmente si existe prótesis mecánica.

- Es preferible frente a los DOAC en caso de falla renal avanzada (TFG menor de 30 y puede emplearse aun si la tasa de filtración glomerular es de 15).

Si el INR es muy inestable $(60 \%$ de las veces por debajo del rango terapéutico), usarán los NOAC.

Existe una falsa creencia -y muy arraigada- que la warfarina tiene antídoto, lo cual ha hecho pensar que es más segura que los DOAC. Esto es una falacia, pues la acción como antídoto de la vitamina $\mathrm{K}$ empieza a las 24 horas de administrada. No es inmediata. Si se requiere revertir su efecto de urgencia, se debe utilizar concentrado complejo de protombina, poco accesible ${ }^{32-34}$.

En pacientes tratados con warfarina, el INR debe ser determinado al menos semanalmente durante la iniciación de la terapia antitrombótica y mínimo mensualmente cuando la anticoagulación es estable (INR en rango) (nivel de evidencia A).

En pacientes con fibrilación auricular no valvular en quienes no se logre mantener un nivel de INR terapéutico con warfarina, se empleará un NOAC (nivel de evidencia C).

\section{Aspirina}

La evidencia de prevención efectiva de ataque cerebrovascular con Aspirina es débil o casi nula y conlleva riesgo potencial de sangrado. Los datos muestran que el riesgo de sangrado mayor o hemorragia intracerebral no se diferencia con los DOAC, especialmente en el anciano ${ }^{14,35}$. Desde el advenimiento de los DOAC el uso de antiplaquetarios (Aspirina o clopidogrel), o la terapia de combinación para la prevención del ataque cerebro-vascular se limita solo para aquellos enfermos que no acepten terapia con DOAC. La combinación es mejor que el ASA, pero el riesgo de sangrado aumenta. El uso de ASA como monoterapia preventiva del ataque cerebro-vascular en pacientes con fibrilación auricular prácticamente está proscrito por todas las Guías.

\section{Aspirina más clopidogrel}

En la actualidad ninguna Guía establece esta combinación como sustituto a la warfarina o a los DOAC en enfermos con fibrilación auricular. El estudio ACTIVE ${ }^{36}$, comparó warfarina vs. Aspirina-clopidogrel en enfermos con fibrilación auricular y concluyó que el tratamiento con clopidogrel más aspirina comparado con aspirina sola, redujo la tasa de eventos vasculares mayores en enfermos de alto riego para ataque cerebro-vascular pero en quienes no se eligió la warfarina. La combinación fue igual a Aspirina sola (con base en datos de metaanálisis previos), pero fue inferior que la warfarina para ataque cerebro-vascular, y el riesgo de sangrado mayor fue más alto. Así, ni el ASA solo ni la combinación ASA más clopidogrel han demostrado beneficio igual o superior a la warfarina o los DOAC.

\section{Conflicto de intereses}

Los autores declaran no tener conflicto de intereses.

\section{Bibliografía}

1. Camm AJ, Lip GY, De Caterina R, et al. 2012 focused update of the ESC Guidelines for the management of atrial fibrillation: an update of the 2010 ESC Guidelines for the management of atrial fibrillation-developed with the special contribution of the European Heart Rhythm Association. Europace. 2012;14:1385-413.

2. Olesen JB, Fauchier L, Lane DA, et al. Risk factors for stroke and thromboembolism in relation to age among patients with atrial fibrillation: the Loire Valley Atrial Fibrillation Project. Chest. 2012;141:147-53.

3. Olesen JB, Lip GY, Lane DA, et al. Vascular disease and stroke risk in atrial fibrillation: a nationwide cohort study. Am J Med. 2012;125, 826 e13-23.

4. Kaw D, Malhotra D. Platelet dysfunction and end-stage renal disease. Semin Dial. 2006;19:317-22.

5. Gage BF, Waterman AD, Shannon W, et al. Validation of clinical classification schemes for predicting stroke: results from the National Registry of Atrial Fibrillation. JAMA. 2001;285:2864-70.

6. Olesen JB, Lip GY, Hansen ML, et al. Validation of risk stratification schemes for predicting stroke and thromboembolism in patients with atrial fibrillation: nationwide cohort study. BMJ. 2011;342:d124.

7. January CT, Wann LS, Alpert JS, et al. 2014 AHA/ACC/HRS guideline for the management of patients with atrial fibrillation: executive summary: a report of the American College of Cardiology/American Heart Association Task Force on practice guidelines and the Heart Rhythm Society. Circulation. 2014;130:2071-104.

8. Apostolakis S, Sullivan RM, Olshansky B, et al. Factors affecting quality of anticoagulation control among patients with 
atrial fibrillation on warfarin: the SAMe-TT(2)R(2) score. Chest. 2013;144:1555-63.

9. You JJ, Singer DE, Howard PA, et al. Antithrombotic therapy for atrial fibrillation: Antithrombotic Therapy and Prevention of Thrombosis, $9^{\text {th }}$. ed: American College of Chest Physicians Evidence-Based Clinical Practice Guidelines. Chest. 2012;141 2 Suppl, e531S-75S.

10. Potpara TS, Polovina MM, Licina MM, et al. Reliable identification of "truly low" thromboembolic risk in patients initially diagnosed with "lone" atrial fibrillation: the Belgrade atrial fibrillation study. Circ Arrhythm Electrophysiol. 2012;5:319-26.

11. Olesen JB, Torp-Pedersen C, Hansen ML, et al. The value of the CHA2DS2-VASC score for refining stroke risk stratification in patients with atrial fibrillation with a CHADS2 score 0-1: a nationwide cohort study. Thromb Haemost. 2012;107:1172-9.

12. Van Staa TP, Setakis E, Di Tanna GL, et al. A comparison of risk stratification schemes for stroke in 79,884 atrial fibrillation patients in general practice. J Thromb Haemost. 2011;9:39-48.

13. Abu-Assi E, Otero-Ravina F, Allut Vidal G, et al. Comparison of the reliability and validity of four contemporary risk stratification schemes to predict thromboembolism in nonanticoagulated patients with atrial fibrillation. Int J Cardiol. 2013;166:205-9.

14. Friberg L, Rosenqvist M, Lip GY. Evaluation of risk stratification schemes for ischaemic stroke and bleeding in 182678 patients with atrial fibrillation: the Swedish Atrial Fibrillation cohort study. Eur Heart J. 2012;33:1500-10.

15. Boriani G, Botto GL, Padeletti L, et al. Improving stroke risk stratification using the CHADS2 and CHA2DS2-VASC risk scores in patients with paroxysmal atrial fibrillation by continuous arrhythmia burden monitoring. Stroke. 2011;42:1768-70.

16. Siu CW. One more " C" for CHA2DS2-VASC score? J Am Coll Cardiol. 2015;65:1602-3.

17. Chao TF, Lin YJ, Tsao HM, et al. $\operatorname{CHADS}(2)$ and $\mathrm{CHA}(2) \mathrm{DS}(2)$ VASC scores in the prediction of clinical outcomes in patients with atrial fibrillation after catheter ablation. J Am Coll Cardiol. 2011;58:2380-5.

18. NICE. Atrial Fibrillation. Quality Standard. National Institute for Health and Clinical Excellence; 2015.

19. Verma A, Cairns JA, Mitchell LB, et al. 2014 focused update of the Canadian Cardiovascular Society Guidelines for the management of atrial fibrillation. Can J Cardiol. 2014;30:1114-30.

20. Gage BF, Yan Y, Milligan PE, et al. Clinical classification schemes for predicting hemorrhage: results from the National Registry of Atrial Fibrillation (NRAF). Am Heart J. 2006;151:713-9.

21. Pisters R, Lane DA, Nieuwlaat R, et al. A novel user-friendly score (HAS-BLED) to assess 1-year risk of major bleeding in patients with atrial fibrillation: the Euro Heart Survey. Chest. 2010;138:1093-100.

22. Fang MC, Go AS, Chang Y, et al. A new risk scheme to predict warfarin-associated hemorrhage: The ATRIA (Anticoagulation and Risk Factors in Atrial Fibrillation) Study. J Am Coll Cardiol. 2011;58:395-401.

23. Lip GY, Andreotti F, Fauchier L, et al. Bleeding risk assessment and management in atrial fibrillation patients. Executive Summary of a Position Document from the European Heart Rhythm
Association [EHRA], endorsed by the European Society of Cardiology [ESC] Working Group on Thrombosis. Thromb Haemost. 2011;106:997-1011.

24. Roldan V, Marin F, Fernandez $\mathrm{H}$, et al. Predictive value of the HAS-BLED and ATRIA bleeding scores for the risk of serious bleeding in a "real-world" population with atrial fibrillation receiving anticoagulant therapy. Chest. 2013;143: 179-84.

25. Apostolakis S, Lane DA, Guo Y, et al. Performance of the HEMORR(2)HAGES, ATRIA, and HAS-BLED bleeding riskprediction scores in patients with atrial fibrillation undergoing anticoagulation: the AMADEUS (evaluating the use of SR 34006 compared to warfarin or acenocoumarol in patients with atrial fibrillation) study. J Am Coll Cardiol. 2012;60:861-7.

26. Lip GY, Frison L, Halperin JL, Lane DA. Comparative validation of a novel risk score for predicting bleeding risk in anticoagulated patients with atrial fibrillation: the HAS-BLED (Hypertension, Abnormal Renal/Liver Function, Stroke, Bleeding History or Predisposition, Labile INR, Elderly, Drugs/Alcohol Concomitantly) score. J Am Coll Cardiol. 2011;57:173-80.

27. Gallego P, Roldan V, Torregrosa JM, et al. Relation of the HAS-BLED bleeding risk score to major bleeding, cardiovascular events, and mortality in anticoagulated patients with atrial fibrillation. Circ Arrhythm Electrophysiol. 2012;5: 312-8.

28. Olesen JB, Lip GY, Lindhardsen J, et al. Risks of thromboembolism and bleeding with thromboprophylaxis in patients with atrial fibrillation: A net clinical benefit analysis using a 'real world' nationwide cohort study. Thromb Haemost. 2011;106:739-49.

29. Friberg L, Rosenqvist M, Lip GY. Net clinical benefit of warfarin in patients with atrial fibrillation: a report from the Swedish atrial fibrillation cohort study. Circulation. 2012;125: 2298-307.

30. Hirsh J, Fuster V, Ansell J, Halperin JL. American Heart Association/American College of Cardiology/American College of Cardiology Foundation guide to warfarin therapy. J Am Coll Cardiol. 2003;41:1633-52.

31. Tung JM, Mamdani MM, Juurlink DN, et al. Rates of ischemic stroke during warfarin treatment for atrial fibrillation. Stroke. 2015;46:1120-2.

32. Wiedermann CJ, Stockner I. Warfarin-induced bleeding complications clinical presentation and therapeutic options. Thromb Res. 2008;122 Suppl2:S13-8.

33. Makris M, Watson HG. The management of coumarininduced over-anticoagulation Annotation. $\mathrm{Br} \mathrm{J}$ Haematol. 2001;114:271-80.

34. Hanley JP. Warfarin reversal. J Clin Pathol. 2004;57:1132-9.

35. Mant J, Hobbs FD, Fletcher K, et al. Warfarin versus aspirin for stroke prevention in an elderly community population with atrial fibrillation (the Birmingham Atrial Fibrillation Treatment of the Aged Study, BAFTA): a randomised controlled trial. Lancet. 2007;370:493-503.

36. Investigators A, Connolly SJ, Pogue J, et al. Effect of clopidogrel added to aspirin in patients with atrial fibrillation. N Engl J Med. 2009;360:2066-78. 